INPLASY

PROTOCOL

To cite: Fu et al. The Efficacy of Baduanjin Exercise for Parkinson's Disease: A Protocol of Systematic Review and Meta-analysis. Inplasy protocol 202210093. doi: 10.37766/inplasy2022.1.0093

Received: 18 January 2022

Published: 18 January 2022

Corresponding author:

Fu Qizhan

1077939879@139.com

Author Affiliation:

Shaanxi University of Chinese Medicine.

Support: This study was not funded.

Review Stage at time of this submission: The review has not yet started.

Conflicts of interest:

None declared.

\section{The Efficacy of Baduanjin Exercise for Parkinson's Disease: A Protocol of Systematic Review and Meta-analysis}

Fu, Q1'; Zhao, Z2; Du, H3; Liu, M4; Han, Z5.

Review question / Objective: There are many exercise therapies in the treatment of Parkinson's disease, such as yoga, taichi, qi dance. etc. However, there is still a lack of evidence on the improvement effect of Baduanjin exercise on Parkinson's disease. Therefore, the aim of this study is to explore whether Baduanjin exercise has certain effectiveness in relieving symptoms of Parkinson's disease. Some questions will be included: whether Baduanjin exercise has more advantages in improving motor symptoms of Parkinson's disease; whether Baduanjin exercise is more effective in improving non-motor symptoms of Parkinson's disease compared with other exercise therapies.

Condition being studied: Nowadays, Baduanjin exercise is effective for many diseases, such as low back pain, stroke rehabilitation and so on.However, the efficacy of Baduanjin exercise to improve the quality of life of Parkinson's disease is better than other therapies, there is still a lack of evidence for systematic reviews and meta-analysis.

INPLASY registration number: This protocol was registered with the International Platform of Registered Systematic Review and Meta-Analysis Protocols (INPLASY) on 18 January 2022 and was last updated on 18 January 2022 (registration number INPLASY202210093).

\section{INTRODUCTION}

Review question / Objective: There are many exercise therapies in the treatment of Parkinson's disease, such as yoga, taichi, qi dance. etc. However, there is still a lack of evidence on the improvement effect of Baduanjin exercise on Parkinson's disease. Therefore, the aim of this study is to explore whether Baduanjin exercise has certain effectiveness in relieving symptoms of Parkinson's disease. Some questions will be included: whether Baduanjin exercise has more advantages in improving motor symptoms of Parkinson's disease; whether Baduanjin exercise is more effective in improving non-motor 
symptoms of Parkinson's disease compared with other exercise therapies.

Condition being studied: Nowadays, Baduanjin exercise is effective for many diseases, such as low back pain, stroke rehabilitation and so on. However, the efficacy of Baduanjin exercise to improve the quality of life of Parkinson's disease is better than other therapies, there is still a lack of evidence for systematic reviews and meta-analysis.

\section{METHODS}

Participant or population: We will include studies that patients diagnosed as Parkinson's Disease, not limited by age, sex, race, nationality. Patients should have been diagnosed with Parkinson's Disease based on past orcurrent guidelines for the diagnosis of Parkinson's Disease.

Intervention: The patients of the experimental group are taking Baduanjin exercise alone, or in combination with another active treatment, such as balance pad training, ear acupoint pressure pills. etc.

Comparator: There is no limits on intervention in the control group. With the exception of Baduanjin exercise,the intervention of the control group mainly include conventional western medicine treatment.

Study designs to be included: The included studies are randomized clinical trials (RCTs).

Eligibility criteria: (1) randomized controlled trials;(2) peer-reviewed studies in English or Chinese; (3) Baduanjin Qigong as the main intervention in the studies.

Information sources: The PubMed, Web of Science, Embase, Cochrane Central Register of Controlled Trials, Clinical Trials.gov, Cochrane Library, SinoMed, Chinese National Knowledge Infrastructure Database and Wan Fang database will be searched from inception to January 2022 to identify potentially eligible studies.
Main outcome(s): The primary outcome measures are Movement Disorder SocietyUnified Parkinson's Disease Rating ScalePart III(MDS-UPDRS-III), Six-Minute Walk Test (6MWT), Hamilton Depression Scale(HAMD), Hamilton Anxiety Scale(HAMA),Berg Balance Scale (BBS).

Quality assessment / Risk of bias analysis: Two of our reviewers will independently use the risk of bias tool provided by the Cochrane Collaboration to evaluate the quality of the included studies. If there is disagreement in the assessment, it will be discussed with the third reviewer.

Strategy of data synthesis: Review Manager5.4 (RevMan 5.4) provided by the Cochrane Collaboration Network will be used for data analysis. The mean difference or standardized mean difference and $95 \%$ Cls will be utilized to calculate continuous outcome data. The heterogeneity of included studies will be assessed by Qand 12 test statistics. As for $Q$ statistics, if $P>0.051$ orl $2<50 \%$, we will use a fixed effect model for meta-analysis; if $\mathrm{P} 50 \%$, we will analyze data using a random-effects model.

Subgroup analysis: We will investigate the source of heterogeneity using subgroup analysis based on different interventions, controls, and outcomes.

Sensitivity analysis: In order to evaluate the sensitivity of the meta-analysis, studies will be conducted to exclude one by one and analyze the source of heterogeneity.

\section{Country(ies) involved: China.}

Keywords: Baduanjin exercise; Parkinson's Disease;meta-analysis.

Contributions of each author:

Author 1 - Fu Qizhan.

Author 2 - Zhao Zhengtai.

Author 3 - Du Hong.

Author 4 - Liu Mingyu.

Author 5 - Han Zucheng. 